\title{
A STUDY OF THE MENSTRUAL CYCLE IN $M A C A C A$ IRUS WITH SPECIAL REFERENCE TO THE DETECTION OF OVULATION
}

\author{
C. J. MAHONEY \\ Department of Dental Science, Royal College of Surgeons of England, \\ Downe, Orpington, Kent \\ (Received 15th May 1969)
}

\begin{abstract}
Summary. Three methods were used for the detection of ovulation in twenty-five cycles of sixteen monkeys of the species Macaca irus: (1) rectal palpation of the urogenital tract, (2) the karyopycnotic index (KPI) of the vaginal smear cytology, and (3) a simple chemical test for the apparent changes in the chloride content of the vaginal mucus.

Daily vaginal smears were necessary to indicate the first day of the menstrual cycle as, in some animals, menstruation began as an occult flow lasting 1 to 3 days. Rectal palpation of the uterus and ovaries gave the precise time of ovulation and was the only method which would differentiate, with certainty, between ovulation and anovulation.

Both KPI and the vaginal mucus chloride test failed to indicate accurately the time of ovulation, and in some cases gave a false indication of ovulation, but the chloride test did give several days' warning that ovulation was imminent.
\end{abstract}

\section{INTRODUCTION}

Investigation of the physiological changes occurring in the reproductive tract during the menstrual cycle of the monkey is not only of importance to those interested in breeding these animals but may supply useful information for comparative human studies, for example in the study and detection of ovulation.

Apart from pregnancy, direct viewing of the ovaries by laparoscopy and recovery of tubal ova are, at present, the only ways of proving, beyond doubt, that ovulation has occurred in the human. In the monkey, Macaca mulatta, changes in size and consistency of the uterus and ovaries assessed by rectal palpation were used by Hartman $(1932,1933)$ to determine accurately the time of ovulation.

The percentage of exfoliated vaginal epithelial cells containing pycnotic nuclei has been used to indicate the time of ovulation in man and other species (Papanicolaou, 1933, 1946; De Allende, Shorr \& Hartman, 1945).

A simple chemical test was devised by McSweeney \& Sbarra (1964) which appeared to measure daily changes in the chloride ion concentration of human vaginal mucus. It was later concluded that variations in the chloride concentration were only apparent and that the test indicated, indirectly, the quantity of 
mucus secreted by the cervical glands (McSweeney \& Sbarra, 1965). They compared the results of this technique with the fern test (Papanicolaou, 1946; Rydeberg, 1948; Campos da Paz, 1951), spinnbarkeit (Clift, 1945; Cohen, Stein \& Kaye, 1952) and the cornification index of the vaginal smear cytology (Papanicolaou, 1933). A modification of this method was adapted by Hardy, Lewis, Little \& Swyer (1970) and comparison was made with the basal body temperature shift (Rubenstein, 1937; Riley, Dontas \& Gill, 1955) and the karyopycnotic index of the vaginal smear in human volunteers.

In the present investigation, an attempt has been made to correlate the cyclical changes in the karyopycnotic index and the apparent variation in the chloride concentration of the vaginal mucus with the palpable physical changes in the urinogenital tract of the monkey.

\section{MATERIALS AND METHODS}

Sixteen female monkeys of the species Macaca irus, aged $3 \frac{1}{2}$ to 8 years and weighing between 3.6 and $6 \mathrm{~kg}$, were used in this study. A total of twenty-five cycles was followed using three techniques for the detection of ovulation. Daily observations were made during sixteen cycles. Less frequent observations were made during the remaining nine cycles because of the difficulty of carrying out all three techniques every day. The three methods employed to detect ovulation are described below:

(1) The ovaries, uterus and cervix were examined rectally to determine changes in the physical characteristics of each organ during the menstrual cycle. While the assessment of ovarian size by rectal palpation is necessarily only an approximation, comparison can be made with the many ovaries obtained at post mortem during various phases of the menstrual cycle. In the early follicular or late luteal phase, ovaries measured approximately $7 \times 6 \times 5$ $\mathrm{mm}$. During the mid-follicular phase, ovaries measured approximately $9 \times 8 \times 5$ $\mathrm{mm}$. An ovary containing a ripe Graafian follicle would have twice the volume of an ovary at the beginning of the menstrual cycle. On this basis, therefore, ovaries were classified, on rectal palpation, as small, medium, or large.

(2) Using a modified Papanicolaou staining technique, the cyclical variation in the karyopycnotic index of the vaginal smear cytology was studied. Under phase contrast microscopy, pycnotic nuclei appear as bright red bodies (Wied, 1955). One hundred superficial and intermediate epithelial cells were counted on two slides and the percentage of the total containing pycnotic nuclei was expressed as the karyopycnotic index (KPI).

(3) A simple test was used for the approximate estimation of the apparent changes in cervical mucus chloride concentration during the cycle. Vaginal mucus was applied to a test paper impregnated with silver chromate as described by Hardy $e t$ al. (1970). Chloride ions in the mucus are precipitated as silver chloride. When serial dilutions of saline ranging from 0.1 to $1.0 \% \mathrm{NaCl}$ were applied to the chloride-sensitive paper, spot intensities equivalent to those given by the mucus during the cycle, were produced. Apparent chloride concentrations of the vaginal mucus were therefore expressed as the equivalent $\mathrm{NaCl}$ solution concentration. 
Observations were carried out as follows: the monkeys were sedated by an intramuscular injection of phencyclidine $\mathrm{HCl}$ (Sernylan, Parke-Davis \& Co.). Following thorough cleansing, the vulva and lower vagina were dilated with a pharyngeal dilator to permit the introduction of a cotton applicator. A sample of mucus and cells was removed from the upper vagina and posterior fornix of the cervix. The applicator was lightly applied to a microscope slide and the smear was immediately fixed. The applicator, still carrying moist material, was then applied to the chloride-sensitive test paper.

Daily sedation of animals was not always possible and vaginal smears were taken from these monkeys through the mesh of the cage. Rectal palpation was performed under sedation at less frequent but regular intervals during the cycle.

\section{RESULTS}

From observations made on thirty monkeys over a period of 1 year, it was found that monkeys could be divided into three groups according to the onset and duration of blood flow during the menses.

(a) Menstrual flow began abruptly and was overt from the outset in the majority of animals. External signs of menstruation lasted 1 to 6 days, although a 3-day period was the commonest. Following the overt phase, no external signs of bleeding were visible but the presence of a considerable volume of blood in the vagina was demonstrable by vaginal swabbing for a period of 1 to 3 days. This was defined as occult uterine bleeding.

(b) Occult uterine bleeding heralded menstruation in a smaller number of monkeys. Overt flow then ensued and persisted for a further 1 to 3 days. Of the twenty-five cycles in the present study, two cycles exhibited these menstrual characteristics.

(c) A few monkeys rarely showed overt signs of menstruation yet the subjects were proved, by close observation, to have regular ovulatory cycles. As Day 1 of the cycle is regarded as the first day of menstrual bleeding, daily vaginal smears were necessary to detect traces of blood in the tract in order to establish the beginning of the cycle.

Eighteen of the twenty-five cycles investigated proved, by rectal palpation, to be ovulatory. An ovulatory cycle was defined as one in which the active ovary underwent a gradual increase in size during the follicular phase followed by a sudden reduction in size at ovulation. No sudden reduction in size occurred in anovulatory cycles. The mean length of the follicular phase in seventeen cycles was $14 \cdot 1$ days (S.D. $\pm 3 \cdot 1$ ) and the mean luteal period was 17.3 days (S.D. $\pm 5 \cdot 8)$. The total length of the remaining cycle was 11 days with a follicular phase of 6 days. As this would seem to be a rare type of cycle, it was not included in the above calculations.

\section{OVULATORY CYGLES}

\section{Rectal palpation of the urogenital tract}

Cervix uteri. No significant change in size or consistency of the cervix was found during the ovulatory menstrual cycles. 
Uterus. The body of the uterus was hard or firm in every animal on Day 1 of the cycle. It remained firm for some days and then a progressive resilient change set in until, at mid-cycle, the uterus was either distinctly resilient or, in one case, soft. The mean period of resilience was 1.8 days (S.D. \pm 0.7 ). Uterine resilience was detectable on the day of ovulation in every cycle, and for the 1 or 2 days preceding ovulation in some subjects. On the day following ovulation, the uterus lost its resilience and became firm again.

In three ovulatory cycles, the uterus became slightly resilient for 1 day during the mid-follicular phase.

A second period of uterine resilience, not as marked as that occurring earlier,

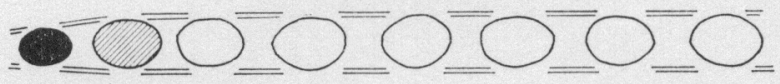

Right ovary
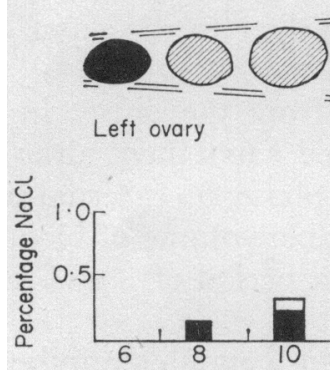

Left ovary

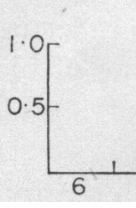

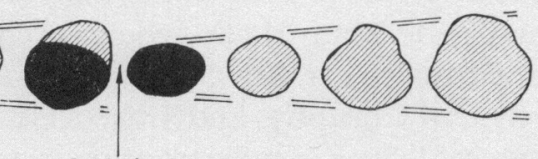

Ovulation

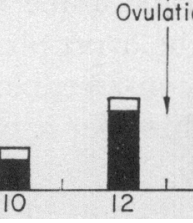

Day of cycle
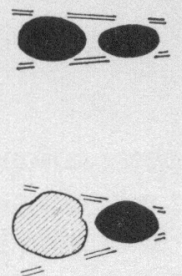

Ovarian consistency

- Firm

0 Resilient

Soft or resilient

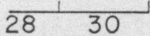

TeXT-FIG. 1. Ovulatory cycle in Macaca irus. Correlation between changes in physical characteristics of both ovaries during the cycle in one individual monkey with the apparent variation in the chloride concentration of the vaginal mucus expressed as the equivalent $\mathrm{NaCl}$ solution concentration. Ovulation occurred on Day 13. Progressive ovarian regression occurred between Day 20 and 28.

developed during the luteal phase of six cycles, and persisted for 1 day only. It occurred on average, 4.5 days (S.D. \pm 1.7 ) after ovulation.

Ovaries. Both ovaries underwent physical changes during the menstrual cycle. In all but three of the eighteen cycles studied, the growth of one ovary was a continuously progressive change leading up to ovulation. The results obtained from one individual monkey are presented as representative of the ovulatory cycles (Text-fig. 1).

During the first 7 or 8 days of the cycle, both ovaries were approximately the same size, shape and firm consistency. Although both gonads then increased in size to approximately the same extent and became resilient, it was impossible at this stage to predict which ovary would eventually bear a ripe follicle.

By the 10th day, one ovary had increased markedly in size and resilience and it was possible to predict that the follicle would develop in this ovary. The opposite gonad had enlarged only slightly and was less resilient than previously. The developing ovary continued to enlarge until, by Day 12, the follicle became palpable. By Day 13, the ovary had diminished to half its former size, ovulation having occurred during the previous $24 \mathrm{hr}$. 
On four occasions, dehiscence of the follicle was felt while palpating the ovary. The sensation was of a large, thin-walled vesicle under high pressure suddenly rupturing. Within $5 \mathrm{sec}$, the ovary had decreased to half its former size and was distinctly firmer in consistency. Ovulation may have been precipitated by the act of palpating the ovary, but dehiscence probably did not occur much before the physiologically ripe moment.

During the 6 days following ovulation, the ovary enlarged again and became resilient, reaching a maximal size on Day 20; this secondary growth of the ovary was associated with the second period of uterine resilience. A slight. irregularity of the ovarian surface indicated the position of the fully developed corpus luteum. However, in many cases it was not possible to identify the corpus. luteum although a pin-head-sized protuberance was occasionally palpable on the ovarian surface. Presumably this was the stigma formed at ovulation. Over the remainder of the cycle, the ovary diminished in size and became firm until, by the onset of the next menses, both ovaries were approximately the same small size.

The rate at which an active ovary developed and underwent the changes just described varied from one monkey to another, but the changes were continuous and progressive. In three cycles, however, the active ovary displayed an erratic growth. Cessation of growth, lasting 1 or 2 days, occurred at a stage of advanced follicular development and, in addition, a moderate resilience developed transiently in the uterus. The ovary then resumed its growth and ovulation occurred some days later. Marked resilience developed in the uterus at the time of ovulation.

\section{Karyopycnotic index (KPI)}

The KPI reached its first peak on the last day of overt flow or during the following occult menstrual phase, i.e. on Day 3 or 4 .

A secondary peak in the KPI was associated with the presence of an enlarged ovary and ovulation, but there seemed to be no constant relationship between the peak and the day of ovulation (Text-fig. 2a, b). The summit of the curve occurred from 3 days before to 3 days after ovulation and in only two cycles did the peak develop on the day of ovulation.

A tertiary peak in the KPI was found in seven of the eighteen cycles occurring at a mean of $5 \cdot 3$ days (S.D. $\pm 2 \cdot 2$ ) after ovulation. It coincided, approximately, with the peak of secondary maximal growth of the ovulated ovary, at a time when the corpus luteum was presumably most active. During many of the cycles studied, numerous small peaks in the KPI developed during the luteal phase, but none could be related to any physical change in the reproductive tract.

\section{Mucus chloride spot test}

During the early follicular phase, little chloride was demonstrable in the vaginal mucus. At a time when both ovaries began to increase in size, the mucus spot intensities were equivalent to an $\mathrm{NaCl}$ concentration of $0.3 \%$. The intensity increased above the $0.3 \%$ level at a time when it was apparent in which ovary the mature follicle was destined to develop. There was a sudden increase in intensity of the spot to above the $0.5 \%$ level occurring at a mean of 3.5 days 

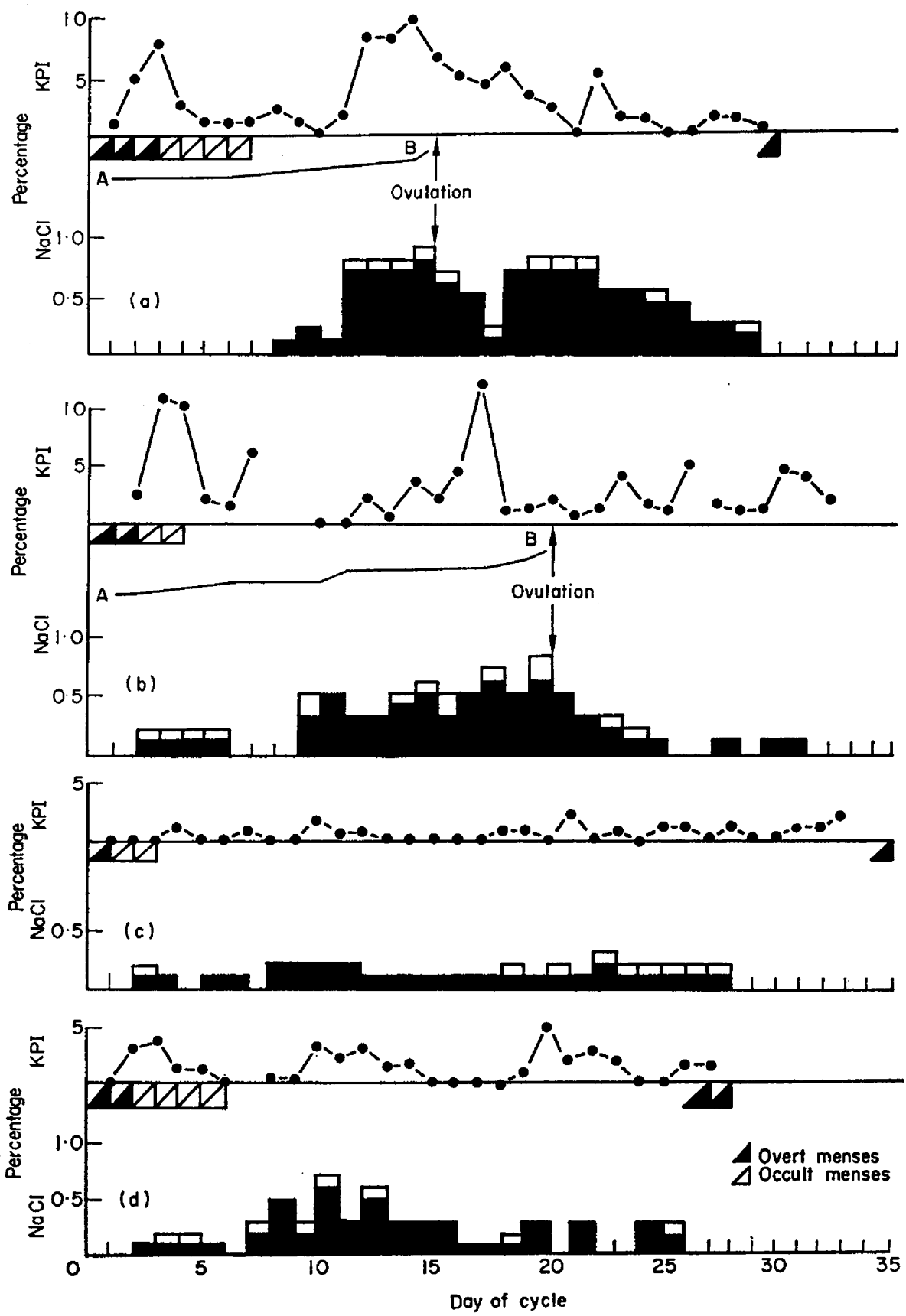

TexT-FIG. 2. (a to d) Correlation between the KPI of the vaginal smear and the apparent variation in the chloride concentration of the vaginal mucus expressed as the equivalent NaCl solution concentrations during the cycle of Macaca irus. Open bars indicate a range in the spot intensity due to the difficulty, on occasions, of designating the equivalent concentration exactly. (a) Ovulatory cycle. The line AB, with arbitrary base-line, represents the continuously progressive growth of the active ovary leading to ovulation. (b) Ovulatory cycle. The line AB represents erratic growth of the active ovary leading to ovulation. Two phases of arrested development occurred. No smear was taken on Days 8, 9 and 27. Graph discontinued after Day 32 (cycle length 39 days). (c) Anovulatory cycle. Minimal ovarian development was exhibited. The KPI is of a low plateau type. (d) Anovulatory cycle. Marked ovarian development was displayed. The KPI is similar to an ovulatory type. 
(S.D. $\pm 0 \cdot 8$ ) preceding ovulation; this level was maintained for 1.3 days (S.D. \pm $0 \cdot 8$ ) after ovulation. A maximally intense spot was found on 1 day in only three cycles. Only in one subject did the brightest spot develop on the day of ovulation.

A second period of bright spots developed during the luteal phase in five of the eighteen cycles studied. The spots were of intensities equal to those found during the primary period in some cases, but in others $0.5 \% \mathrm{NaCl}$ equivalent concentration was the maximum attained.

No secondary period of intense yellow spots developed in the remaining thirteen cycles studied. After the peak around mid-cycle, the intensity of the spots fell to $0.3 \%$ and below, and persisted at this level for the remainder of the cycle.

\section{ANOVULATORY GYCLES}

Although seven of the twenty-five cycles studied proved to be anovulatory, there was great variation in the degree of ovarian development.

\section{Rectal palpation of the urogenital tract}

Cervix uteri. Again, no significant change in the physical characteristics of the cervix was detectable during any of the anovulatory cycles.

Uterus. A slight resilience, which persisted for 1 or 2 days, was detectable in the uterus at mid-cycle in the four most extreme anovulatory cycles studied. Resilience did not develop during the second half of the cycles. In two subjects, where ovarian development was more pronounced, the uterus exhibited a moderate degree of resilience at mid-cycle, which lasted for 2 and 3 days in each subject. Slight uterine resilience persisting for 6 days was evident at mid-cycle in the last member of the group.

Ovaries. Practically no development of the ovaries was apparent in one cycle and resilient changes at mid-cycle were slight. The ovaries began to develop normally during the first half of the cycle in three animals but by Day 9 or 10 regressive changes took place. At no stage was a developing follicle palpable.

In the remaining three anovulatory cycles, steady growth in one ovary was obvious from the beginning of the cycle up till Day 9 or 10 when the gonad reached a large size and was distinctly resilient. At this stage, an enlarged follicle was palpable in the ovary. No further growth in the ovary took place after this point. The texture of the ovary changed suddenly to a distinct firmness on Day 11 in two animals. It remained in this condition until Day 19 or 20 when the ovary again transiently became resilient.

In the third subject, the large follicle remained resilient for 6 days during which time the uterus retained a slight resilience. The ovary then regressed and became firm. These cycles were deemed to be anovulatory as no sudden reduction in size of the enlarged active ovary occurred, as was evident in ovulatory cycles following ovulation.

Karyopycnotic index $(K P I)$

A low plateau type of KPI was found in the cycle displaying the most extreme dysfunction of the ovaries (Text-fig. 2c). During the phase of uterine bleeding in 
three cycles with less severe ovarian hypofunction, a peak comparable to that found in ovulatory cycles occurred. The KPI began to rise in the post-menstrual and early follicular phase of these cycles but declined as further ovarian development ceased. During the remainder of these cycles, the KPI had a low undulating pattern.

In two cycles showing marked ovarian development, the KPI was basically similar to that seen in ovulatory cycles (Text-fig. 2d). A well-defined peak formed during menstruation and at mid-cycle, and a smaller peak developed mid-way through the second half of one cycle. No record was made of the KPI in the remaining anovular cycle.

\section{Mucus chloride spot test}

The mucus spots were uniformly dull during the whole of the cycle exhibiting minimal ovarian activity (Text-fig. 2c). During the first few days of the three cycles demonstrating less severe ovarian dysfunction, the spots attained intensities equivalent to 0.3 to $0.5 \% \mathrm{NaCl}$ solution. Once the ovaries ceased their growth, the mucus spots diminished in intensity.

A pattern of spots identical to that found in ovulatory cycles developed in two of the cycles demonstrating marked ovarian activity. The spots remained bright for 4 or 5 days at mid-cycle and in one cycle, a second period of intense spots developed transiently half way through the second half of the cycle (Text-fig. 2d).

In the remaining non-ovulatory cycle, the mucus spots attained an intensity equivalent to a $1.0 \%$ solution of $\mathrm{NaCl}$. This high level persisted for 6 days during which period the uterus maintained a slightly resilient texture and a persistent resilient follicle was palpable in the ovary. As the ovary began to regress, the intensity of the spots diminished suddenly and remained faint throughout the remainder of the cycle.

Menstrual dates over a 3-year period have been recorded for all the animals used in this study. Calculation of the mean and range of cycle lengths indicated that the manipulation involved in rectal palpation and vaginal swabbing did not in itself modify the cycle length.

\section{DISCUSSION}

Ovulation in Macaca irus occurs between Days 12 and 17 of the cycle (Fujiwara \& Imamichi, 1966). It was recommended by these workers that the male and female should be caged together for mating for 1 week from the 11 th day of the cycle. Day 1 of the cycle is taken as the first day of menstrual bleeding. As the results presented indicate, the first day of overt menstruation is not necessarily the first day of the cycle. An occult phase, lasting 1 to 3 days, may precede the overt flow and unless daily vaginal smears are taken to detect traces of blood in the tract, a miscalculation of up to 3 days could be made in assessing the first day of the cycle. This could be critical for a fertile mating as ovulation may have occurred $48 \mathrm{hr}$ before cageing the male and female together on what appeared to be 11 th day of the cycle.

In describing his techniques of bimanual rectal palpation of the reproductive 
tract of Macaca mulatta, Hartman $(1932,1933)$ noted that the uterine body, which is firm at the beginning of the cycle, softened only when an enlarged ovary was present. Although growth changes in the uterus and ovaries were evident during anovulatory cycles, he found that uterine softening did not develop at mid-cycle.

The results obtained from the study of the menstrual cycle in Macaca irus broadly support Hartman's findings. Distinct resilient changes in the uterus occurred at mid-cycle only when an enlarged follicle was present in the ovary. This was so even when the follicle failed to rupture in anovulatory cycles. In cycles showing extreme ovarian hypofunction, little or no uterine resilience developed at mid-cycle. On the day following ovulation in every cycle studied, the uterus had lost its resilience. It would seem, therefore, that ovarian hormones are responsible for the consistency changes in the uterus and that, with the collapse of the follicle at ovulation, these hormones (probably oestrogens) are lost to the blood stream. Further evidence in support of this view is supplied by the marked ovarian enlargement displayed in some anovulatory cycles when a large resilient follicle was palpable in some instances. At a time when one would have expected ovulation to occur, the enlarged ovary suddenly lost its resilient texture, became firm but failed to undergo the sharp reduction in size exhibited during ovulatory cycles. At the same time, the uterus lost its resilience. The KPI and the mucus spot test falsely indicated that ovulation had occurred. It would seem that the enlarged follicle, in failing to rupture, became luteinized and began to secrete progestagens.

During one of the anovular cycles studied, an enlarged follicle developed and persisted for 6 days without losing its resilient texture. The uterus also remained moderately resilient and the intense mucus spots indicated a prolonged hyperoestrogenism. It was not until the ovary suddenly became firm at the end of this period that the uterus lost its resilience and the intensity of the mucus spots declined.

Growth of the ovary during the follicular phase is not always a continuously progressive enlargement. In three ovulatory cycles, after a few days of growth there was a lag before the ovary recommenced its enlargement. One of the reasons for ovulation occurring unusually late in the cycle may be a temporary disruption of the pituitary-ovarian axis resulting from some unidentified influence.

Cyclical changes in the cornification index of the exfoliate vaginal cytology were studied during the menstrual cycle in Macaca mulatta (De Allende, Shorr \& Hartman, 1945). A peak at mid-cycle was associated with ovulation. In a smaller number of cases, a peak of lower amplitude developed during the luteal phase and was related to the presence of a mature corpus luteum. This pattern was evident in the present study of Macaca irus. However, in all the ovulatory cycles and in all but the most extreme hypofunctional anovular cycles, a welldefined peak developed during the menstrual phase on Day 3 or 4. A menstrual peak in the KPI was noted in the human by De Allende et al. (1945), Pundel (1950) and Fukushima, Stevens, Gantt \& Vorys (1964). It seems unlikely that oestrogens are responsible for the nuclear pycnosis of the vaginal epithelium at this time as rectal palpation of the ovaries in the monkey during menstruation 
suggests that the gonads are relatively quiescent and there is little follicular development.

De Allende et al. (1945) suggested that exfoliation of the deeper layer of the vaginal epithelium may occur during menstruation, increasing the number of pycnotic intermediate cells in the smear. Derangements of the blood supply to the vagina may occur at this time with consequent tissue hypoxia.

In their study of human subjects, Hardy et al. (1970) assumed that the summit of the mid-cycle KPI indicated the point of ovulation. No constant relationship between the peak of the KPI and the day of ovulation was apparent in the cycle of Macaca irus. The peak occurred from 3 days before to 3 days after ovulation.

Available evidence suggests that circulating oestrogenic hormones stimulate the cervical mucus glands to increase their secretion. Bergman \& Lund (1951) showed that the mucus of the human vagina remains approximately isotonic throughout the menstrual cycle. The total amount of $\mathrm{NaCl}$ in the dried mucus was shown to rise sharply at mid-cycle by Herzberg, Joel \& Katchalsky (1964). They found that the water content of fresh mucus increased at this time as a result of which the osmotic pressure of the mucus was maintained. As McSweeney \& Sbarra (1965) pointed out, the spot test does not really indicate changes in chloride ion concentration of the cervical mucus but reflects the quantity of mucus produced by the cervical glands.

During the follicular phase of the human menstrual cycle, mucus producing spot intensities equivalent to $0 \cdot 3 \% \mathrm{NaCl}$, or above, were regarded by $\mathrm{McSw}$ weeney \& Sbarra (1965) as being favourable to sperm penetration. They designated this 'the fertile phase'. It is interesting to note that, during the ovulatory cycles studied in Macaca irus, one ovary began to show marked enlargement from the time when the mucus spots reached and exceeded the $0.3 \% \mathrm{NaCl}$ equivalent. Before this time, it was impossible to predict in which ovary a Graafian follicle would eventually develop.

The chloride spot test did not indicate accurately the moment of ovulation but it did give several days' warning that ovulation was about to take place. It gave a false indication of ovulation in some anovulatory cycles, showing only slight depression of ovarian function, and this possibility should be borne in mind when cycles in humans are being studied.

Hardy et al. (1970) found a high degree of correlation between the first day of the cycle on which the mucus spots exceeded the equivalent of $0.5 \% \mathrm{NaCl}$ and the total length of the cycle in man, and between the former and the length of the luteal phase. Their results indicated that the luteal phase of the cycle is not constant. In the relatively few cycles of Macaca irus examined, the results similarly suggested that both the follicular and luteal phases are variable. These observations are in agreement with De Allende et al. (1945) in their study of Macaca mulatta.

Rectal palpation of the genital organs yields a great deal of information pertaining to ovarian development during the menstrual cycle in monkeys. It is the only technique of the three methods described which differentiates with certainty between ovulatory and non-ovulatory cycles and can determine precisely the moment of ovulation. 


\section{ACKNOWLEDGMENTS}

I am grateful to Mr N. R. Hardy, Miss L. Lewis, Dr V. Little and Dr G. I. M. Swyer of the Endocrine Unit of University College Hospital Medical School, for supplying me with the chloride-sensitive test papers and to my colleagues at the Royal College of Surgeons of England for their help in the preparation of this paper.

\section{REFERENCES}

Bergman, P. \& Lund, C. G. (1951) Osmotic pressure of human cervical mucus: cyclic changes in osmotic pressure of cervical mucus and effect of osmotic changes on spermatozoal motility. Acta obstet. gynec. scand. 30, 267.

CAMPos DA PAZ, A. (1951) Studies on crystallization of cervical mucus and its relationship to cervical receptivity to spermatozoa. Am. F. Obstet. Gynec. (Suppl.) 61A, 790.

Cuift, A. F. (1945) Observations on certain rheological properties of human cervical secretions. Proc. R. Soc. Med. 39, 1.

Cohen, M. R., SteIn, I. F. \& KaYe, B. M. (1952) Spinnbarkeit: A characteristic of cervical mucus. Fert. Steril. 3, 201.

De Allende, I.L.C., Shorr, E. \& Hartman, C. G. (1945) A comparative study of the vaginal smear cycle of the rhesus monkey and human. Contr. Embryol. 31, 1.

Fujiwara, T. \& IMAMICHI, T. (1966) Breeding of cynamolgus monkeys as an experimental animal. Fap. F. med. Sci. Biol. 19, 215.

Fuxushima, M., Stevens, V. C., GantT, C. L. \& Vorys, N. (1964) Urinary Fsh and lh excretion during the normal menstrual cycle. F. clin. Endocr. Metab. 24, 205.

Hardy, N. R., Lewis, L., LitTLe, V. \& Swyer, G. I. M. (1970) Use of a spot test for chloride in cervical mucus for self-detection of the fertile phase in women. F. Reprod. Fert. $21,143$.

Hartman, C. G. (1932) Studies in the reproduction of the monkey Macacus (Pithecus) rhesus, with special reference to menstruation and pregnancy. Contr. Embryol. 23, 1.

Hartman, C. G. (1933) Pelvic (rectal) palpation of the female monkey with special reference to the ascertainment of ovulation time. Am. 7. Obstet. Gynec. 26, 600.

Herzberg, M., Joel, C. A. \& Katchalsky, A. (1964) The cyclical variation of sodium chloride content in the mucus of the cervix uteri. Fert. Steril. 15, 684 .

McSweEney, D. J. \& Sbarra, A. J. (1964) A new cervical mucus test for hormone appraisal. Am. $\mathcal{J}$. Obstet. Gynec. 88, 705.

McSwerney, D. J. \& Sbarra, A. J. (1965) A rapid ovarian hormone and ovulation test. Obstet. Gynec., N.Y. 26, 201.

Papantcolaov, G. N. (1933) The sexual cycle in the human female as revealed by vaginal smears. Am. J. Anat. 52, 519.

Papanicolaou, G. N. (1946) General survey of vaginal smear and its use in research diagnosis. Am. $\mathcal{F}$. Obstet. Gynec. 51, 316.

Pundel, J. P. (1950) Les frottis vaginaux et cervicaux. Masson, Paris.

Riley, G. M., DontAs, E. \& GiLL, B. (1955) Use of serial vaginal smears in detecting time of ovulation. Fert. Steril. 6, 86.

Rubenstein, B. B. (1937) The relation of cyclic changes in human vaginal smears to body temperature and basal metabolic rates. Am. F. Physiol. 119, 635.

RYDEBERG, E. (1948) Observations on the crystallization of cervical mucus. Acta obstet. gynec. scand. 28, 172.

WIED, G. L. (1955) Suggested standard for karyopyknosis. Use in hormonal reading of vaginal smears. Fert. Steril. 6, 61 . 